\title{
Xuebijing injection attenuates pulmonary injury by reducing oxidative stress and proinflammatory damage in rats with heat stroke
}

\author{
YI CHEN $^{1}$, HUASHENG TONG ${ }^{2}$, ZHIGUO PAN ${ }^{2}$, DONGXIN JIANG ${ }^{1}$, \\ XINQIN ZHANG ${ }^{2}$, JUNMING QIU ${ }^{2}$, LEI SU $^{2}$ and MINZHOU ZHANG ${ }^{3}$ \\ ${ }^{1}$ Department of Critical Care Medicine, The Fifth People's Hospital of Dongguan, \\ Affiliated to Medical College of Jinan University, Dongguan, Guangdong 523900; \\ ${ }^{2}$ Department of Critical Care Medicine, Guangzhou General Hospital of Guangzhou Military Command, \\ Key Laboratory of Tropical Trauma Care and Tissue Repair of PLA, Guangzhou, Guangdong 515000; \\ ${ }^{3}$ Department of Critical Care Medicine, Guangdong Hospital of Traditional Chinese Medicine, \\ Guangzhou, Guangdong 510120, P.R. China
}

Received October 8, 2015; Accepted January 26, 2017

DOI: $10.3892 /$ etm.2017.4444

\begin{abstract}
The present study aimed to investigate the protective effect of Xuebijing injection (XBJ) on lung injury in heat-stroke rats and the underlying mechanisms. In total, 54 rats were randomly assigned to non-thermal, saline vehicle and XBJ groups. The rectal temperature (Tc), mean arterial pressure (MAP) and respiratory rate (RR) of the rats were recorded. The time-point of heat stroke and the time of survival were assessed, and indicators of arterial blood gas were regularly measured from 0 to $60 \mathrm{~min}$. The concentration of tumor necrosis factor- $\alpha$ (TNF- $\alpha$ ), interleukin- $1 \beta$ (IL- $1 \beta)$ and IL-10 was also determined. At the end of the experiment, lung tissue was harvested for histopathological analysis. Inducible nitric oxide synthase (iNOS) and superoxide dismutase (SOD) expression was measured by immunohistochemistry. Terminal deoxynucleotidyl transferase-mediated dUTP nick-end labeling was used to measure apoptosis. XBJ pretreatment prolonged the decline of clinical characteristics, as demonstrated by increases in Tc, MAP, RR and indicators in arterial blood gas in rats under heat stress. The time until heat stroke
\end{abstract}

Correspondence to: Professor Lei Su, Department of Critical Care Medicine, Guangzhou General Hospital of Guangzhou Military Command, Key Laboratory of Tropical Trauma Care and Tissue Repair of PLA, 111 Liuhua Road, Guangzhou, Guangdong 515000, P.R. China

E-mail: slei_icu@163.com

Professor Minzhou Zhang, Department of Critical Care Medicine, Guangdong Hospital of Traditional Chinese Medicine, 111 Dade Road, Guangzhou, Guangdong 510120, P.R. China

E-mail: minzzhang@yeah.net

Key words: Xuebijing injection, heat stroke, pulmonary injury, oxidative stress, inflammation, apoptosis and the survival time in the Saline group were shorter than in rats treated with XBJ. The expression of iNOS in lung tissue and the concentration of TNF- $\alpha$, IL- $1 \beta$ and IL-10 in the bronchoalveolar lavage fluid of rats treated with saline was higher than in rats with XBJ pre-treatment. Contrarily, SOD expression in rats treated with saline was decreased compared with that in rats treated with XBJ. Moreover, the apoptotic rate in the lung tissues of rats with saline treatment was higher than that in rats treated with XBJ. In conclusion, XBJ delayed the development of heat stroke and increased the survival time in rats under heat-stress by ameliorating pulmonary failure and acute lung injury. The underlying mechanisms of this effect may be the reduction of inflammatory cytokines as well as attenuation of oxidative stress and apoptosis by XBJ.

\section{Introduction}

Heat stroke is a physical dysfunction characterized by a high core temperature $\left(>40^{\circ} \mathrm{C}\right)$, hypovolemia, coma and convulsions. It is usually induced by heat stress and tends to result in high mortality and deformity (1). To date, the underlying mechanisms of heat stroke have remained elusive. However, evidence has indicated that the pathophysiological changes of heat stroke include dysfunction of thermal regulation, abnormal expression of heat stroke protein, destruction of the intestinal barrier and activation of coagulation (1-5). Despite symptomatic treatment and rapid cooling, these changes always lead to lethal systemic inflammatory response syndrome (SIRS) or multiple organ dysfunction syndromes (MODS) (2). The key mediators of heat stress activate the cascade of proinflammatory cytokines and the initiation of oxidative stress (6).

Xuebijing injection (XBJ) is a Chinese patent drug composed of Honghua (Carthamus tinctorius), Chi shao (Paeoniae radix), Danshen (Salvia divinorum), Danggui (Angelica sinensis) and Chuanxiong (Ligusticum wallichii Franchet). It was approved for use in the treatment of sepsis in 2004 (7). In the past decades, XBJ has been proved to 
alleviate inflammation, reduce oxidative stress, regulate immunity and improve coagulation in clinical practice as well as in experimental studies (8). In a study focusing on liver ischemia/reperfusion, XBJ was observed to exert a protective effect by reducing the production of malondialdehyde (MDA), increasing the level of superoxide dismutase (SOD) and attenuating apoptosis in the liver (9). In addition, XBJ has been shown to alleviate pulmonary inflammation and reduce SIRS caused by pneumonia or sepsis $(10,11)$. Since pathophysiological changes induced by heat stroke are similar to changes caused by sepsis (1), it was speculated that XBJ pre-treatment may protect the lungs of rats from heat stress. The present study assessed this and further explored the underlying mechanisms of this effect and its correlation with inflammatory and oxidative injury.

\section{Materials and methods}

Animals and materials. Since estrogen has a definite effect on organ injury induced by heat stroke (12), only male animals were used in the present study. A total of 54 adult male Sprague Dawley rats [weight, 220-260 g; SCXK (Yue) 2006-0015 no. 4402100446] were purchased from the Animal Experiment Center of Southern Medical University (Guangzhou, China). XBJ injections were purchased from Tianjin Chase Sun Pharmaceutical Co. Ltd (Tianjin, China). All experimental procedures were approved by the Animal Care and Use Committee of Guangzhou General Hospital of Guangzhou Millitary Command prior to the study.

Grouping and administration. Rats were initially housed for $72 \mathrm{~h}$ at an ambient temperature of $25 \pm 0.5^{\circ} \mathrm{C}$ with a humidity of $35 \pm 5 \%$. Food and tap water were available ad libitum. A total of 54 rats were randomly divided into three groups: Saline pre-treatment heat stroke group (Saline group, $n=18$ ), XBJ pre-treatment heat stroke group (XBJ group, $n=18$ ) and non-thermal group (Sham group, $n=18$ ). Rats in the XBJ group were injected with $\mathrm{XBJ}$ ( $4 \mathrm{ml} / \mathrm{kg}$, two times per day) through the tail vein for three days before the experiment $(13,14)$. In the Sham and Saline groups, rats were injected with phosphate-buffered saline for three days $(4 \mathrm{ml} / \mathrm{kg}$, two times a day) prior to the experiment. All rats were included in the analysis of changes in arterial blood gas. However, only 6 rats in each group were utilized to observe changes in mean arterial pressure (MAP), rectal temperature (Tc), the time until heat stroke and the survival time. The remaining 12 rats in each group were used to harvest bronchoalveolar lavage fluid (BALF) and lung tissue for testing inflammatory cytokines, cellular apoptosis as well as the expression of SOD and iNOS at $60 \mathrm{~min}$ in the experiment. The animals were randomly assigned to the subgroups.

Heat stroke model. Intraperitoneal injection of sodium pentobarbital (50 mg/kg; Sigma-Aldrick; Merck KGaA, Darmstadt, Germany) was performed to anesthetize the rats and abolish the corneal and pain reflexes. A femoral arterial catheter was inserted to monitor MAP by a multi-parameter physiological monitor (Vista; Dräger, Lübeck, Germany); Tc and the respiratory rate $(\mathrm{RR})$ were also detected continuously. Rats were placed in a pre-warmed chamber (temperature, $40.0 \pm 0.5^{\circ} \mathrm{C}$; humidity, 60\%) and when the MAP dropped from the peak to $25 \mathrm{mmHg}$ with a $\mathrm{Tc}$ of $>42^{\circ} \mathrm{C}$, the heat-stroke model was considered to be constructed successfully (15). After $1 \mathrm{~h}$, 12 rats from each group were sacrificed with an overdose of pentobarbital $(200 \mathrm{mg} / \mathrm{kg})$ and used for harvesting samples. The remaining rats were observed until dead in the chamber.

Arterial blood gas analysis. A pilot study by our group showed that the formation of heat stroke in the saline-treated rats was $73.6 \pm 1.8 \mathrm{~min}$ under heat stress (16) similar to the time determined by previous studies $(17,18)$. Therefore, arterial blood $(0.5 \mathrm{ml})$ was drawn from the right femoral artery at 0,20 , 40 and $60 \mathrm{~min}$ after the start of the experiment. All samples were measured using a blood gas analyzer (GEM 3000; Instrumentation Laboratory, Bedford, MA, USA) according to the manufacturer's instructions. The partial pressures of oxygen $\left(\mathrm{PaO}_{2}\right)$ and carbon dioxide $\left(\mathrm{PaCO}_{2}\right)$ as well as lactic acid values (Lac) were recorded.

Investigation of survival time. Eighteen rats were used to observe physiological changes and survival time under continuous heat stress until they died. The survival time of animals in the XBJ and Saline groups were investigated. Sham rats were observed at $25^{\circ} \mathrm{C}$ temperature until they recovered from anesthesia.

BALF collection and pulmonary pathological analysis. Following sacrifice with an overdose of sodium pentobarbital at $60 \mathrm{~min}$, the rats were immediately fixed onto a board. The thoracic cavity was opened and the right principal bronchus was ligated. A ' $\mathrm{T}$ ' incision was created in the lower trachea and a venous catheter (16G) was then slowly imbedded. After the establishment of trachea cannula, $3 \mathrm{ml}$ of Saline was infused into the left lung and extracted slowly for 10 times, and the irrigating solution was collected for testing. This process was repeated and the gathered fluid was centrifuged at $800 \mathrm{x}$ g for $10 \mathrm{~min}$. The supernatant was reserved and stored in polypropylene microcentrifuge tubes at $-80^{\circ} \mathrm{C}$. The right lung of each rat was harvested and immersed in $100 \%$ formalin for $24 \mathrm{~h}$, imbedded in paraffin and sectioned. To measure lung injury, three sections of each sample were cut from the base to the apex at 3-mm intervals and stained with hematoxylin and eosin (HE). Ten random regions in every section were scrutinized at x400 magnification (BX51T; Olympus, Osaka, Japan) by two pathologists blinded to the grouping. Five indicators were selected, including intra-alveolar infiltration of neutrophils, interstitial infiltration of neutrophils, peripheral vascular infiltration of neutrophils, pulmonary congestion, and alveolar hemorrhage, with each indicator graded at four levels separately (0-3) (19). The total scores of each section were calculated and the mean score of three sections was the final histological result of acute lung injury in each rat.

Measurement of inflammatory cytokine concentration in $B A L F$. The concentrations of tumor necrosis factor (TNF- $\alpha$ ), interleukin-1 $\beta$ (IL-1 $\beta$ ) and IL-10 in the BALF were measured using ELISA kits (TNF- $\alpha$ determination kit, cat. no. 27194; IL-1 $\beta$ determination kit, cat. no. 27193; IL-10 determination kit, cat. no. 27195; R\&D Systems, Inc., Minneapolis MN, USA) according to the manufacturer's instructions. Each sample was 
A

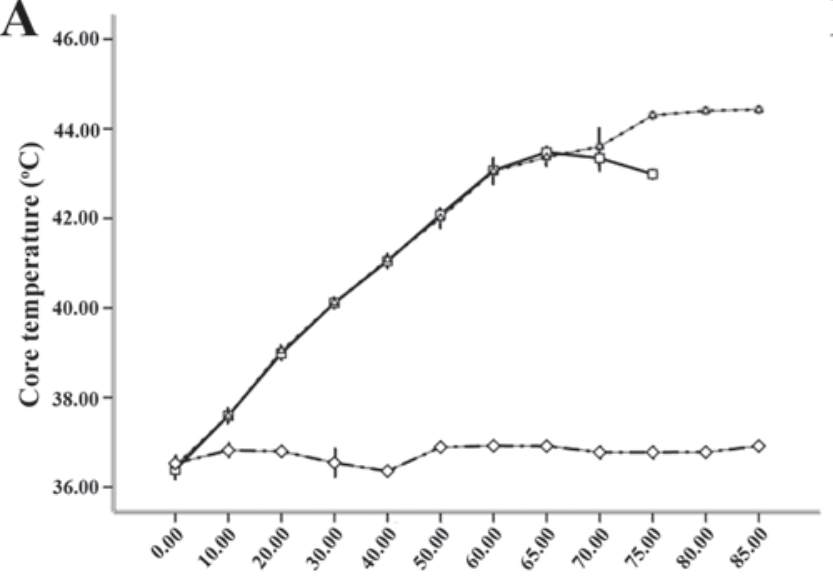

Time (min)

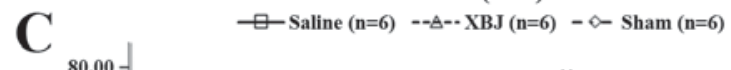

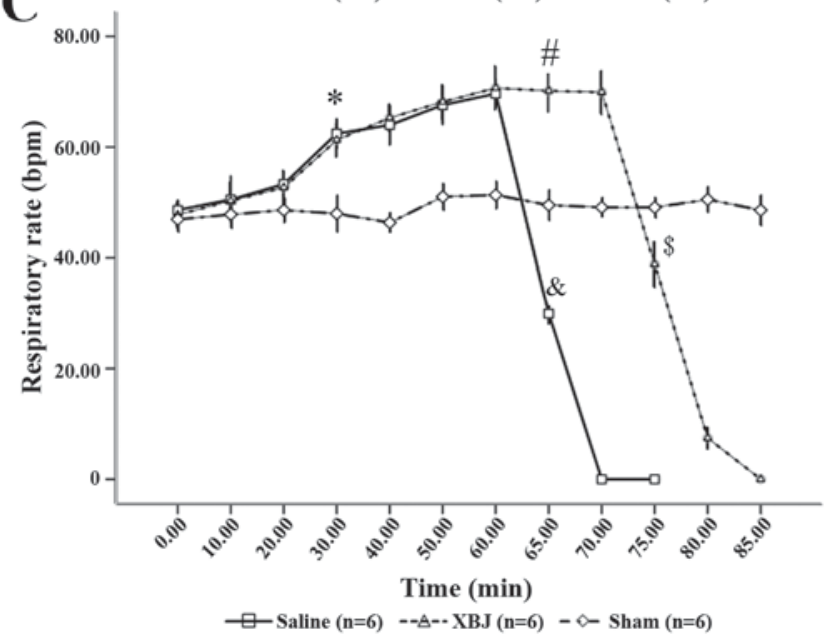

B

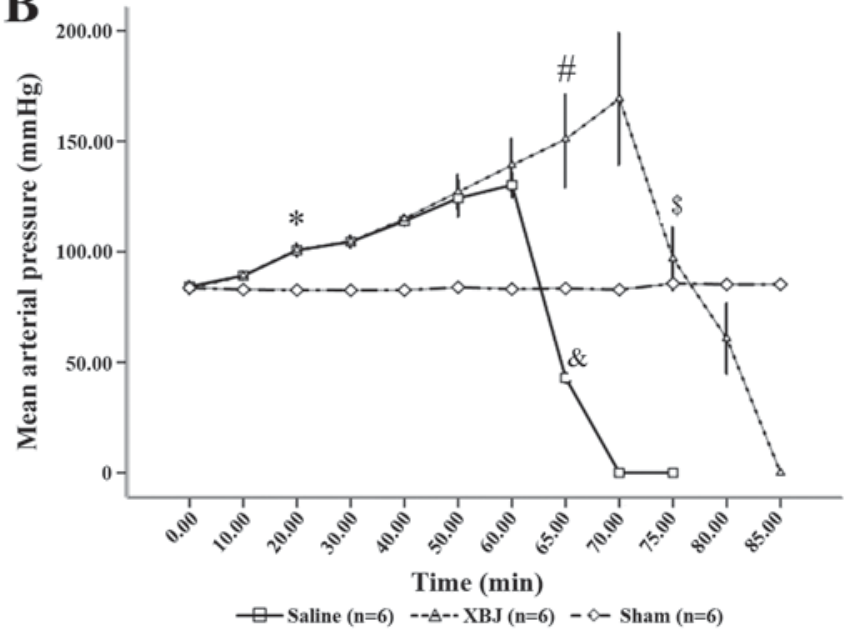

Figure 1. Changes of (A) Tc, (B) MAP and (C) RR in rats. " $\mathrm{P}<0.01$ Saline and XBJ compared with Sham; ${ }^{~} \mathrm{P}<0.01$ compared among the three groups; ${ }^{2} \mathrm{P}<0.01$ compared with Saline $(60 \mathrm{~min}) ;{ }^{\mathrm{S}} \mathrm{P}<0.01$ compared with XBJ $(70 \mathrm{~min})$. Groups: Saline, pre-treated with saline $(4 \mathrm{ml} / \mathrm{kg}$, twice a day for 3 days) and subjected to heat stress; XBJ, pre-treated with Xuebijing injection ( $4 \mathrm{ml} / \mathrm{kg}$, twice a day for 3 days) and subjected to heat stress; Sham, pre-treated with saline (4 ml $/ \mathrm{kg}$, twice a day for 3 days) and kept at ambient temperature.

run in triplicate and the mean was calculated. A plate reader was set at $450 \mathrm{~nm}$ to measure the optical densities (OD) of the samples. According to values of standard OD, a standard curve was displayed to measure the concentration of each sample.

Investigation of oxidative stress and apoptosis. Each lung tissue sample was segmented into three random sections and immunohistochemical analysis was used to detect the expression of inducible nitric oxide synthase (iNOS) and superoxide dismutase (SOD). OD values of ten random fields in each section were measured at x400 magnification (BX51T; Olympus) using Image Pro Plus 6.3 software (Media Cybernetics, Rockville, MD, USA). The comparative optical density (COD) values were determined as the difference between OD values of SOD or iNOS and the background. The mean COD was calculated in each section as the final level of expression of SOD and iNOS.

Terminal deoxynucleotidyl transferase-mediated dUTP nick end labeling (TUNEL) staining was used to detect apoptosis in the lung. Paraffin sections of the lung were stained using the TUNEL technique according to the manufacturer's instructions (TUNEL apoptosis kit, cat. no. C1086; Beyotime Institute of Biotechnology, Haimen, China). The number of apoptotic cells per 100 cells represented the apoptotic rate in each section.

Statistical analysis. Values are expressed as the mean \pm standard deviation and processed using SPSS 17.0 software (SPSS Inc., Chicago, IL, USA). The comparisons of MAP, RR, Tc, arterial blood gas indices and the time until heat stroke in rats were performed by analysis of variance. Comparisons of values between any two groups, including the concentration of inflammatory cytokines in the BALF and pulmonary pathological injury, were performed using the least significant differences (LSD) test or Dunnett's T3 test according to the homogeneity of variance. In certain groups, a paired t-test was used to compare the value at time A to that of time B. Kaplan-Meier analysis was used to display the survival time in the four groups, and a Log-Rank test was used to calculate the difference in median survival time between any two groups. $\mathrm{P}<0.05$ was considered to indicate a statistically significant difference.

\section{Results}

XBJ ameliorates physiological parameters under heat stress. The kinetics of Tc during high-temperature and high-humidity 
Table I. Indicators of arterial blood gas.

Time (min)

\begin{tabular}{|c|c|c|c|c|c|c|}
\hline \multirow[b]{2}{*}{ Indicator } & \multirow[b]{2}{*}{ Group } & & \multirow[b]{2}{*}{ P-value } \\
\hline & & 0 & 20 & 40 & 60 & \\
\hline \multirow[t]{3}{*}{$\mathrm{PaO}_{2}(\mathrm{mmHg})$} & Saline & $86.51 \pm 3.02$ & $88.04 \pm 3.67$ & $92.31 \pm 2.54$ & $57.49 \pm 5.94^{b}$ & \multirow[t]{3}{*}{$<0.01$} \\
\hline & $\mathrm{XBJ}$ & $89.25 \pm 2.32$ & $91.27 \pm 3.48$ & $93.81 \pm 2.46$ & $92.45 \pm 5.62$ & \\
\hline & Sham & $88.64 \pm 2.45$ & $89.23 \pm 4.04$ & $92.82 \pm 4.11$ & $91.43 \pm 2.73$ & \\
\hline \multirow[t]{3}{*}{$\mathrm{PaCO}_{2}(\mathrm{mmHg})$} & Saline & $39.96 \pm 1.82$ & $40.52 \pm 1.46$ & $35.30 \pm 4.59$ & $72.49 \pm 8.96^{\mathrm{b}}$ & \multirow[t]{3}{*}{$<0.01$} \\
\hline & XBJ & $40.49 \pm 2.02$ & $40.59 \pm 2.44$ & $38.47 \pm 3.13$ & $28.24 \pm 3.52^{\mathrm{c}}$ & \\
\hline & Sham & $41.62 \pm 1.83$ & $40.87 \pm 0.92$ & $39.57 \pm 3.63$ & $41.66 \pm 3.45$ & \\
\hline \multirow[t]{3}{*}{$\mathrm{Lac}(\mathrm{mmol} / \mathrm{l})$} & Saline & $0.87 \pm 0.19$ & $1.16 \pm 0.08$ & $2.25 \pm 0.19^{d}$ & $10.17 \pm 3.71^{\mathrm{b}}$ & \multirow[t]{3}{*}{$<0.01$} \\
\hline & XBJ & $0.84 \pm 0.03$ & $1.18 \pm 0.04$ & $2.30 \pm 0.24^{\mathrm{d}}$ & $3.59 \pm 0.22^{\mathrm{c}}$ & \\
\hline & Sham & $0.78 \pm 0.27$ & $1.16 \pm 0.06$ & $1.05 \pm 0.67$ & $0.91 \pm 0.44$ & \\
\hline
\end{tabular}

${ }^{a}$ Comparison among the three groups. ${ }^{b} \mathrm{P}<0.01$ compared with saline $(40 \mathrm{~min}), \mathrm{XBJ}$ (60 min) and Sham (60 min); ${ }^{\mathrm{c}} \mathrm{P}<0.01 \mathrm{compared}$ with Sham (60 min); ${ }^{d} \mathrm{P}<0.01$ compared with Sham $(40 \mathrm{~min})$. Values are expressed as the mean \pm standard deviation ( $\mathrm{n}=18$ per group). Groups: Saline, pre-treated with saline ( $4 \mathrm{ml} / \mathrm{kg}$, twice a day for 3 days) and subjected to heat stress; XBJ, pre-treated with Xuebijing injection (4 ml/kg, twice a day for 3 days) and subjected to heat stress; Sham, pre-treated with saline ( $4 \mathrm{ml} / \mathrm{kg}$, twice a day for 3 days) and kept at ambient temperature. $\mathrm{Pa}$, partial pressure; Lac, lactic acid.

exposure were determined. The Tc of the animals under heat stress increased with time to $42^{\circ} \mathrm{C}$ at $60 \mathrm{~min}$ (Fig. 1A).

There was a significant difference in the changes of MAP among the three treatment groups $(\mathrm{P}<0.01)$. At the start of the experiment, there was no significant difference among the three groups $(\mathrm{P}>0.05)$. However, at $20 \mathrm{~min}$, the values in the Saline and XBJ groups were higher than that in the Sham group $(\mathrm{P}<0.05)$, whereas the values in the Saline and $\mathrm{XBJ}$ groups were comparable $(\mathrm{P}>0.05)$. In the Saline group, the MAP started to decrease significantly after $60 \mathrm{~min}$; at $65 \mathrm{~min}$, the MAP was significantly lower than that in the other two groups $(\mathrm{P}<0.01)$. Similarly, in the XBJ group, the MAP was markedly reduced at $75 \mathrm{~min}$, and the value at $75 \mathrm{~min}$ was significantly lower than that at $70 \mathrm{~min}$ (Fig. 1B; $\mathrm{P}<0.01$ ).

There was no difference in the RR among the three groups at baseline $(\mathrm{P}>0.05)$. The $\mathrm{RR}$ in the Sham group remained stable during the experiment, while the RR in the Saline and XBJ groups was significantly increased after 30 min compared with the Sham group $(\mathrm{P}<0.05)$ More specifically, the value in the Saline and XBJ groups increased from the beginning until 60 min of the experiment and then decreased rapidly in the Saline group, while the value in the XBJ group began to sharply decline at $75 \mathrm{~min}$ (Fig. 1C; $\mathrm{P}<0.01$ between any two groups).

$X B J$ improves heat stroke and survival time. In the Saline group, heat stroke models were successfully generated at $62 \pm 1.5$ min of heat stress. By contrast, models in the XBJ group, the time until heat stroke was $73 \pm 1.2 \mathrm{~min}$, which was significantly longer than that in the Saline-treated rats $(\mathrm{P}<0.01)$.

In the Saline group, the median survival time of the rats from the beginning of experiment was $69 \min$ (95\% CI, 68.04-69.96), which was significantly shorter than the survival time in the XBJ group (82 min; 95\% CI, 79.74-84.26; P<0.01) (Fig. 2).

XBJ stabilizes arterial blood gas in heat-stroke. The $\mathrm{PaO}_{2}$ in the three groups was not significantly changed at the time-points of 0, 20 and $40 \mathrm{~min}$. However, at $60 \mathrm{~min}$, the $\mathrm{PaO}_{2}$ in the Saline group dropped significantly in comparison with that in the other two groups (Table I; $\mathrm{P}<0.01$ ). There was no significant difference between the Sham group and the XBJ group (Table I; $\mathrm{P}>0.05$ ). $\mathrm{PaCO}_{2}$ in the Saline group was significantly higher than that in the $\mathrm{XBJ}$ and Sham groups (Table I; $\mathrm{P}<0.05$ ). Simultaneously, at $60 \mathrm{~min}$, the $\mathrm{PaCO}_{2}$ in the XBJ group was significantly lower than that in the Sham group (Table I; $\mathrm{P}<0.05$ ). In addition, the Lac value in three groups remained stable at the preliminary stage of the experiment. However, at $40 \mathrm{~min}$, the Lac levels in the XBJ and Saline groups were significantly higher than those in the Sham group (Table I; $\mathrm{P}<0.05$ ). At 60 min, the Lac levels in the Saline group were markedly higher than those in the other groups, while those in the XBJ group were also elevated (Table I, $\mathrm{P}<0.01$ ).

XBJ downregulates pulmonary inflammation induced by heat stroke. The concentrations of TNF- $\alpha$, IL-1 $\beta$ and IL-10 in the BALF of rats under heat stress were increased. After $\mathrm{XBJ}$ treatment, the concentrations of these cytokines in the BALF were significantly decreased in comparison with those in the Saline group $(\mathrm{P}<0.01)$. The proportionate variations of TNF- $\alpha$, IL- $1 \beta$ and IL-10 concentrations in BALF were similar (Fig. 3).

XBJ attenuates lung histological injury in heat stroke. As expected, rats in the Saline group showed evidence of alveolar collapse, vascular congestion, alveolar hemorrhage, interstitial thickening, and neutrophil infiltration (Fig. 4A). The pathological injury scores further confirmed that histopathological injury changes in the XBJ group were milder than those in the Saline group, suggesting that XBJ treatment ameliorates the lung damage induced by heat stress (Fig. 4B; $\mathrm{P}<0.01$ ).

XBJ alleviates pulmonary oxidative injury under heat stress. Heat stress significantly increased the expression of SOD 
and iNOS in lung tissues. However, pre-treatment with XBJ ameliorated these changes (Fig. 5).

In the Sham group, there was no obvious expression of SOD, and the structures of the alveoli and mesenchyme were normal (Fig. 5Aa). However, in the Saline group, moderate expression of SOD in the lung was obvious and severe destruction of alveoli was found (Fig. 5Ab). In the XBJ group, the expression of SOD was high on a large scale and the residual structure of the alveoli was integrated (Fig. 5Ac).

In the Sham group, the structure of the alveoli and mesenchyme was normal with no obvious expression of iNOS (Fig. 5Ba). In the Saline group, the expression of iNOS was high with severe destruction of alveoli and infiltration of neutrophils (Fig. 5Bb). In the XBJ group, the expression of iNOS was mild and the structure of the alveoli was clear (Fig. 5Bc).

Apoptotic assay. Following TUNEL staining, apoptotic nuclei showed a dark purple color. The TUNEL staining images are shown in Fig. 6A. In the Sham group, the structure of the alveoli was normal with no obvious apoptotic nuclei. However, in the Saline group, a large number of apoptotic nuclei was evident in the lung, and the lung tissue was destroyed. In the XBJ group, the number of apoptotic nuclei (arrows) was lower compared with that in the Saline group, and in comparison, a normal structure was partially retained.

Quantitative analysis of apoptosis provided the apoptotic rate in each group.Furthermore,XBJ pre-treatment significantly reduced the apoptotic rate induced by heat-stroke (Fig. 6B; $\mathrm{P}<0.01)$.

\section{Discussion}

Circulation, liver, kidney, respiration, the gastro-intestinal tract and coagulation are frequently attacked and damaged by heat stroke, which is the main process for the development of the damage and the cause of death. Understanding the mechanism of the impact heat stroke has on organs is beneficial for the early prevention and treatment and helpful for preventing MODS. Accordingly, it is required to reduce mortality and improve prognosis (20). Clinical manifestations of heat stroke include rhabdomyolysis, circulatory failure, hepatic failure, encephaledema and acute respiratory distress syndrome (ARDS). Approximately $75 \%$ of patients with severe heat stroke developed MODS despite rapid cooling and organ support. Further studies assessed whether respiration is most vulnerable to heat stroke (21). Decades ago, it was shown that an intraperitoneal injection of lipopolysaccharide (LPS) can give rise to severe lung injury (22), and the lungs are always the first organ affected by SIRS. Inflammatory injury following heat stress rather than physical burns is the main cause of lung injury, and diminishing pulmonary inflammation through cooling the whole body ameliorates lung injury (23). According to a previous study by our group, XBJ can alleviate liver injury in heat stroke rats by reducing hepatic inflammation (16). In the present study, the effects of XBJ on pulmonary injury in heat stroke rats and the underlying mechanism were investigated.

Arterial blood gas analysis was used to measure changes of pulmonary function in rats. There was no difference among three groups at baseline. Blood samples were collected at

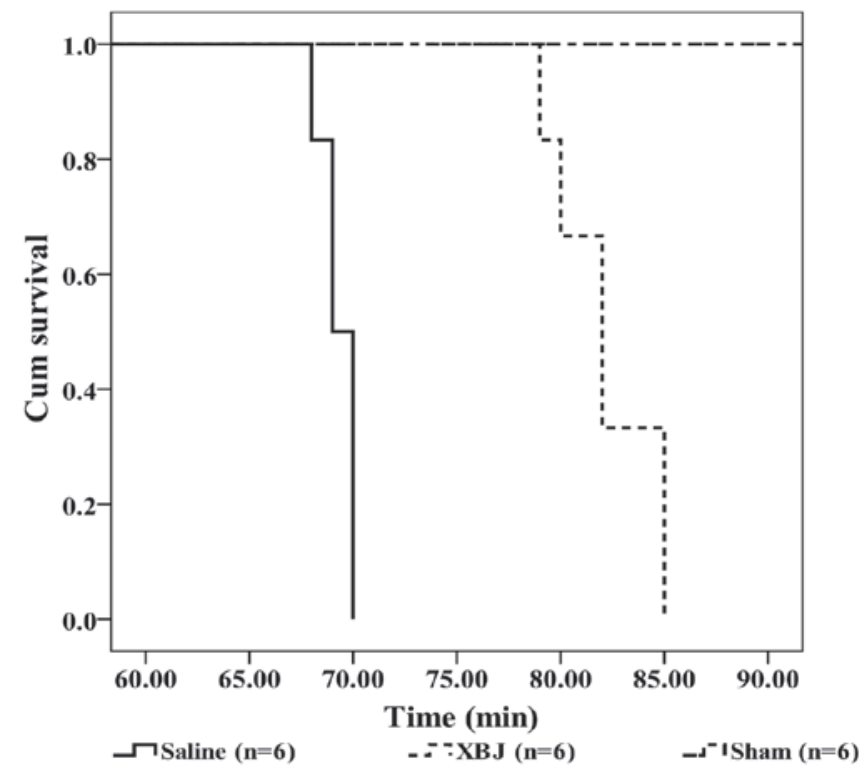

Figure 2. Kaplan-Meier curves showing the survival time of the rats. Groups: Saline, pre-treated with saline ( $4 \mathrm{ml} / \mathrm{kg}$, twice a day for 3 days) and subjected to heat stress; XBJ, pre-treated with Xuebijing injection $(4 \mathrm{ml} / \mathrm{kg}$, twice a day for 3 days) and subjected to heat stress; Sham, pre-treated with saline ( $4 \mathrm{ml} / \mathrm{kg}$, twice a day for 3 days) kept at ambient temperature. $\mathrm{P}<0.01$, Saline compared with XBJ. Cum, cumulative.

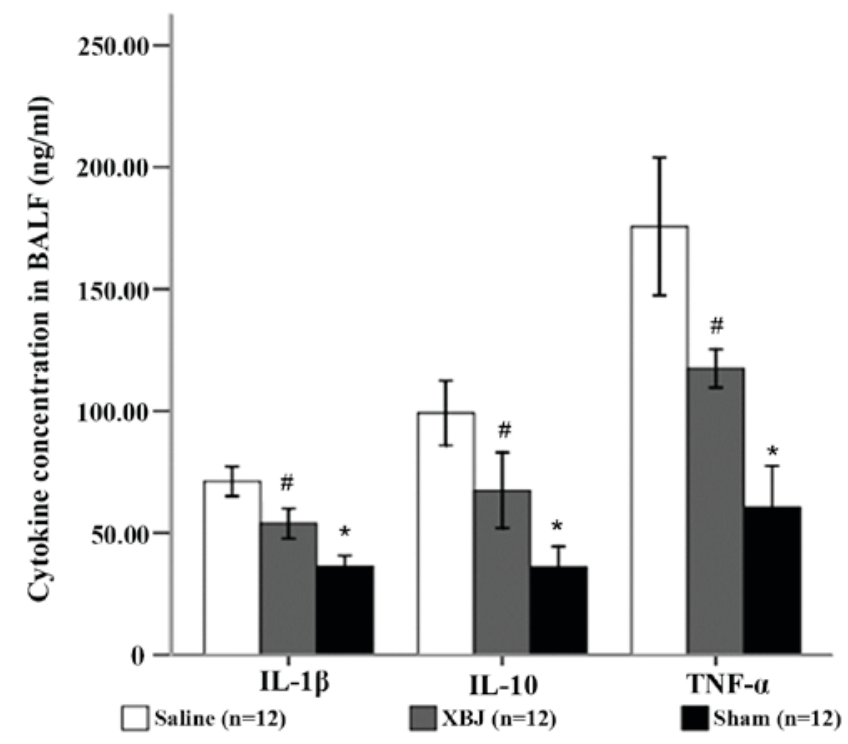

Figure 3. Concentrations of IL-1 $\beta$, IL-10 and TNF- $\alpha$ in BALF. Groups: Saline, pre-treated with saline ( $4 \mathrm{ml} / \mathrm{kg}$, twice a day for 3 days) and subjected to heat stress; XBJ, pre-treated with Xuebijing injection $(4 \mathrm{ml} / \mathrm{kg}$, twice a day for 3 days) and subjected to heat stress; Sham, pre-treated with saline ( $4 \mathrm{ml} / \mathrm{kg}$, twice a day for 3 days) kept at ambient temperature. ${ }^{*} \mathrm{P}<0.05$ compared with Saline; ${ }^{\#} \mathrm{P}<0.01$ compared with sham. BALF, bronchoalveolar lavage fluid; TNF, tumor necrosis factor; IL, interleukin.

three time points $(20,40$ and $60 \mathrm{~min})$ following the start of the experiment. After $40 \mathrm{~min}$, the level of Lac in the groups subjected to heat stress started to increase. It was revealed that the metabolism in rats under heat stress caused an overload of Lac. By contrast, the level of $\mathrm{PaCO}_{2}$ started to decrease, which correlated with the increasing RR triggered by heat stress. Moreover, the augment of RR partly attenuated the acid-base 

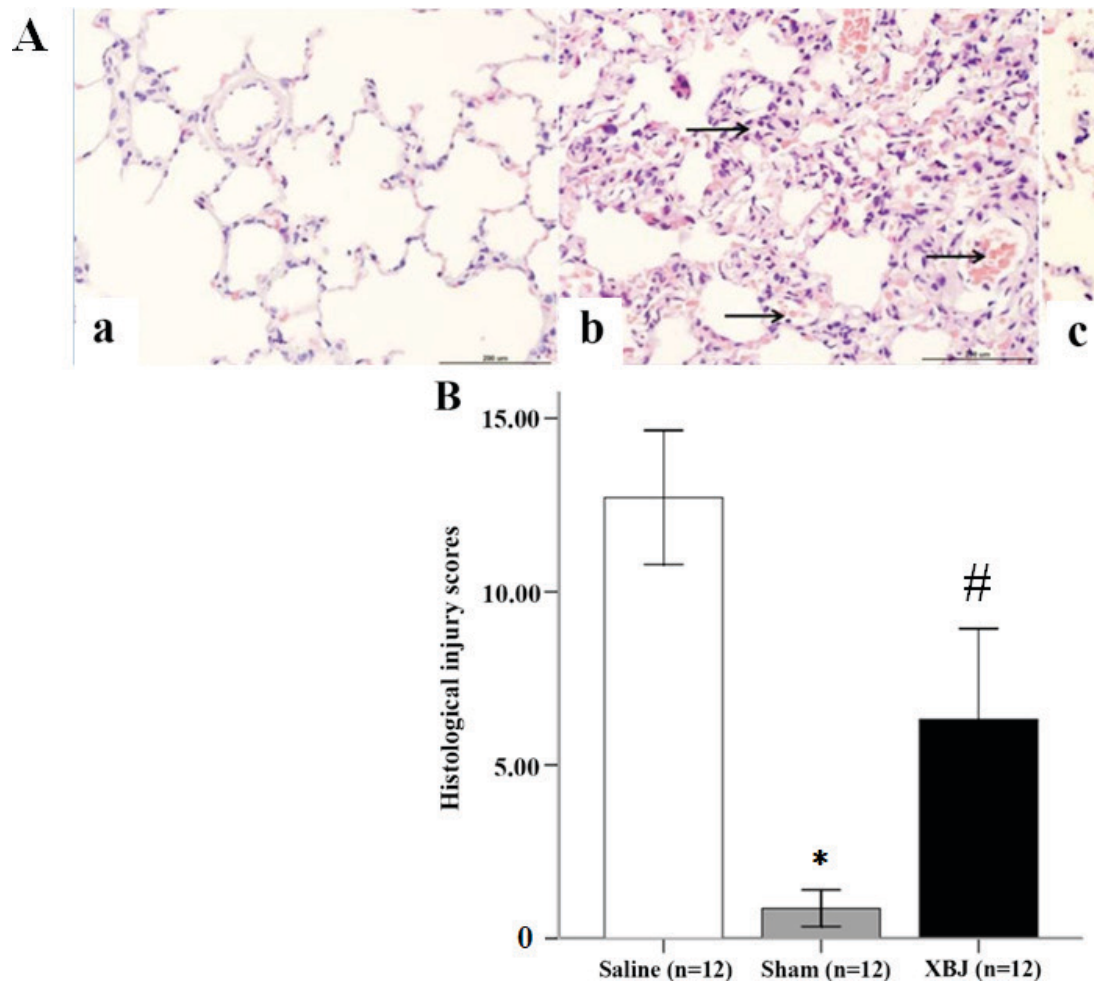

Figure 4. Histological changes and injury scores in rats. (A) Histological changes in the lung tissues of rats (hematoxylin and eosin staining; magnification, x200; scale bar, $200 \mu \mathrm{m}$ ). (a) Sham group. The structure of the alveolus and shape of the mesenchyma were normal, with no evidence of neutrophil infiltration or hemorrhage. (b) Saline group. A large number of neutrophils infiltrated the alveolus and the mesenchyma had an abnormal shape. Alveolar collapse, vascular congestion and alveolar hemorrhage were apparent (arrows). (c) XBJ group. Infiltration of neutrophils, alveolar hemorrhage and interstitial thickening were mild (arrows). (B) Histological injury scores of rats. Groups: Saline, pre-treated with saline (4 ml/kg, twice a day for 3 days) and subjected to heat stress; XBJ, pre-treated with Xuebijing injection ( $4 \mathrm{ml} / \mathrm{kg}$, twice a day for 3 days) and subjected to heat stress; Sham, pre-treated with saline (4 ml/kg, twice a day for 3 days) kept at ambient temperature. ${ }^{*} \mathrm{P}<0.01$ compared with Saline; ${ }^{\#} \mathrm{P}<0.01$ compared with Sham and Saline.

imbalance caused by the increase of Lac induced by heat stress. At $60 \mathrm{~min}$, the $\mathrm{PaO}_{2}$ in the Saline group was lower than that in the other groups. In addition, the $\mathrm{PaO}_{2}$ in the Saline group at $60 \mathrm{~min}$ was lower than that at $40 \mathrm{~min}$. These changes indicated that the compensatory ability of respiration was limited and that of the circulation was exhausted. By contrast, changes of these indicators in the XBJ group were milder than those in the Saline group at $60 \mathrm{~min}$. However, the levels of these indicators in the XBJ group were different to those in the Sham group, such as lower levels of $\mathrm{PaCO}_{2}$ and higher $\mathrm{Lac}$ levels. These differences between the XBJ and Sham group suggested that the respiration and circulation of rats in the XBJ group kept on compensating, and these findings were in accordance with the difference in the formation of heat stroke in rats between XBJ and Saline groups. Therefore, it is concluded that $\mathrm{XBJ}$ improved the tolerance to heat stress so that the failure of respiration was delayed, which may explain the result that the survival time and onset of heat stroke in XBJ group were longer than those in the Saline group obtained in the present study.

TNF- $\alpha$ and IL-1 $\beta$ are important mediators of inflammatory injury and apoptosis $(24,25)$. In patients with ARDS and animals with LPS-induced acute lung injury, the expressions of TNF- $\alpha$ and IL-1 $\beta$ in lung tissue or BALF were significantly increased $(26,27)$. Although IL-10 is an anti-inflammatory cytokine, it was demonstrated that increased IL-10 was accompanied by increases in TNF- $\alpha$ and IL- $1 \beta$ in the local tissue of inflammatory animal models (28). Therefore, the concentration of IL-10 is an indirect marker for assessing the level of systemic inflammation. Measurement of these three cytokines serves as a comprehensive assay for pulmonary inflammation. In the present study, ELISA kits were used to measure the concentrations of the inflammatory cytokines TNF- $\alpha$, IL-1 $\beta$ and IL-10 in BALF, and the results indicated the degree of inflammation in the tissue. The results showed that TNF- $\alpha$, IL- $1 \beta$ and IL-10 in the BALF of the Saline group were increased compared with the XBJ group, which illustrated that the pulmonary inflammation promoted by heat stress was reduced by XBJ treatment. The underlying mechanism of XBJ attenuating pulmonary inflammation may be associated with reducing the overproduction of TNF- $\alpha$, IL-1 $\beta$ and IL-10 in the lung.

H\&E staining and histological injury scores further confirmed that the lung injury in XBJ pre-treated rats was milder than that in the rats under heat stroke without XBJ pre-treatment, which was an objective and direct proof for the preventive effect of XBJ against heat stroke.

SOD is an anti-oxidative protein and treatments stimulating the production of SOD were proved to help to alleviate acute lung injury (29,30). Further studies showed that SOD exerted its protective effects by decreasing the quantity of neutrophiles in the systemic circulation and in alveoli, histological injury and oxidative stress (31). iNOS mainly exists in macrophages, plasmablasts, neutrophils and endothelial cells. It can be activated by TNF- $\alpha$ and IL-1 $\beta$, and produce nitric oxide, which induces lung injury. According to certain studies, the 

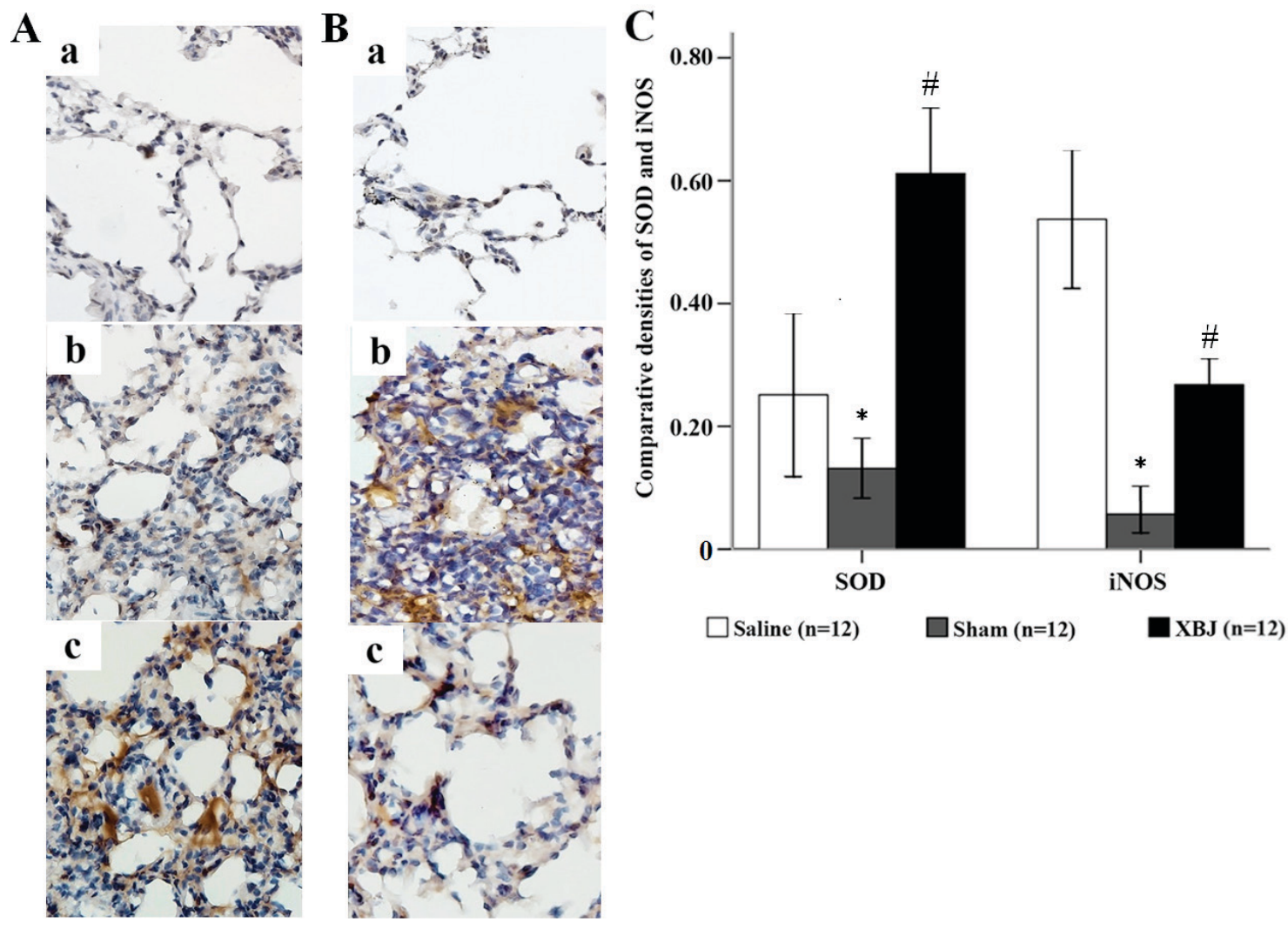

Figure 5. Expression of SOD and iNOS in the lungs of the rats (immunohistochemical staining, magnification, $x 400$ ). (A) Expression of SOD in the lungs of rats. (a) Sham group, (b) Saline group and (c) XBJ group. (B) Expression of iNOS in the lungs of rats. (a) Sham group, (b) Saline group and (c) XBJ group. (C) comparative optical density analysis of SOD and iNOS in the lung. Groups: Saline, pre-treated with saline (4 ml $/ \mathrm{kg}$, twice a day for 3 days) and subjected to heat stress; XBJ, pre-treated with Xuebijing injection ( $4 \mathrm{ml} / \mathrm{kg}$, twice a day for 3 days) and subjected to heat stress; Sham, pre-treated with saline (4 ml $/ \mathrm{kg}$, twice a day for 3 days) kept at ambient temperature. ${ }^{*} \mathrm{P}<0.01$ compared with saline; ${ }^{*} \mathrm{P}<0.01$ compared with sham and Saline. SOD, superoxide dismutase; iNOS, inducible nitric oxide synthase.

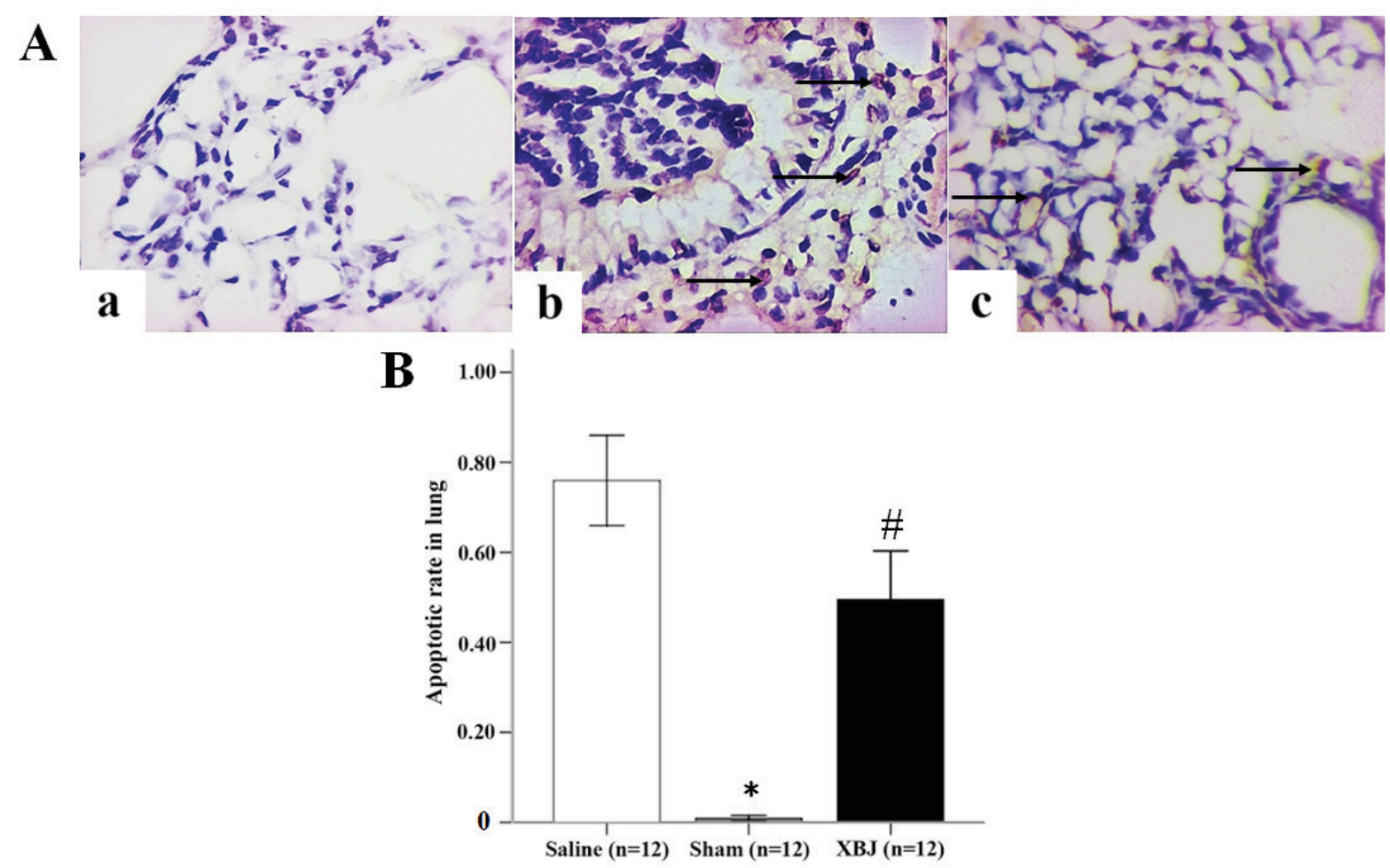

Figure 6. Assessment of apoptosis in the lungs of rats. (A) Apoptotic assay in lung (terminal deoxynucleotidyl transferase-mediated dUTP nick end labeling; magnification, x400). (a) Sham group, (b) Saline group and (c) XBJ group. (B) Apoptotic rate in the lung. Groups: Saline, pre-treated with saline (4 ml $/ \mathrm{kg}$, twice a day for 3 days) and subjected to heat stress; XBJ, pre-treated with Xuebijing injection (4 ml/kg, twice a day for 3 days) and subjected to heat stress; Sham, pre-treated with saline ( $4 \mathrm{ml} / \mathrm{kg}$, twice a day for 3 days) kept at ambient temperature. ${ }^{*} \mathrm{P}<0.01$ compared with Saline; ${ }^{*} \mathrm{P}<0.01$ compared with Sham and Saline. 
primary mechanism of phosgene-induced lung injury is the overproduction of iNOS. Melatonin treatment can attenuate the injury by scavenging radicals and downregulating the production of iNOS (32). Moreover, augmentation of iNOS to induce damage was found in a study on mustard nitrogen-induced lung injury. Aminoguanidine, an inhibitor of iNOS, was shown to ameliorate this injury (33). In the present study, immunohistochemical assays were used to measure the expression of SOD and iNOS in the lungs of rats. Extensive expression of iNOS and little expression of SOD were observed in the rats following saline treatment. Destruction of alveoli and vessels, and infiltration of inflammatory cells were evident. The histological changes and the expression of iNOS in rats with XBJ pre-treatment were significantly lower than those in the Saline group. On the contrary, the levels of SOD in the XBJ group were higher than those in the Saline group. The combination of SOD and iNOS can be regarded as direct indicators representing the level of oxidative stress in the lung. Consequently, XBJ pre-treatment can significantly reduce oxidative stress induced in the lung by heat stress.

In addition, apoptosis in the lung was assessed by using TUNEL staining. The results were used to evaluate pulmonary injury induced by heat stress. Besides inflammation and infiltration of neutrophils, apoptosis was shown to be a typical pathological change in the lungs of rats injected with LPS (34). In particular, apoptosis and damage of alveolar endothelial cells was most frequently seen by TUNEL staining $(35,36)$. Since the physiological function of these cells is disturbed, abnormal alveolar endothelial cells result in failure of oxygenation. Blocking the signaling or upregulating autophagy has been shown to be beneficial to alleviate apoptosis in the lung and attenuate lung injury $(34,37)$. The results of the present study showed that apoptosis in the lungs of rats treated with Saline was more severe than that in the other groups. Therefore, it is concluded that the underlying mechanism of XBJ to reduce lung injury induced by heat stress may be correlated with ameliorating apoptosis of the lung.

Since the time between heat-stroke formation and sacrifice was insufficient in the present study, the effect of XBJ administrated after the preparation was not investigated. Furthermore, no control drug was used due to the absence of an approved medicine for protecting the lungs from heat stress. This may have partly affected the scientific value of the present study. However, the present study still supports the concept that XBJ can ameliorate respiratory failure and attenuate acute lung injury in heat stroke rats. The mechanism may be associated with the inhibition of the overproduction of inflammatory cytokines, reduction of oxidative stress and attenuation of apoptosis. It is therefore suggested that XBJ may be a promising and effective treatment for heat stroke.

\section{Acknowledgements}

This study was supported by the National Natural Science Foundation of China (no. 81503460/81673835/81471839), the Natural Science Foundation of Guangdong Province (no. 2014A030313601), the Technology Planning Project of Guangdong Province (no. 2013B031800010) and the Science and Technology developing Project of Dongguan City (no. 2014108101012).

\section{References}

1. Ji J, Zhou F, Yue H and Song Q: Protective mechanism of Xuebijing injection against heat stroke in rats. Exp Ther Med 7: 1745-1751, 2014.

2. Leon LR, Blaha MD and DuBose DA: Time course of cytokine, corticosterone, and tissue injury responses in mice during heat strain recovery. J Appl Physiol (1985) 100: 1400-1409, 2006.

3. Shevtsov MA, Nikolaev BP, Yakovleva LY, Dobrodumov AV, Dayneko AS, Shmonin AA, Vlasov TD, Melnikova EV, Vilisov AD, Guzhova IV, et al: Neurotherapeutic activity of the recombinant heat shock protein Hsp70 in a model of focal cerebral ischemia in rats. Drug Des Devel Ther 8: 639-650, 2014.

4. Bouchama A, Ollivier V, Roberts G, Al Mohanna F, de Prost D, Eldali A, Saussereau E, El-Sayed R and Chollet-Martin S: Experimental heatstroke in baboon: Analysis of the systemic inflammatory response. Shock 24: 332-335, 2005.

5. Liu Z, Liu JH, Liu Y, Tang Y, Meng F, Sun X, Tang J, Wang JH and Su L: Proteomic analysis and identification of intestinal FBP as a predictor of gut dysfunction during heatstroke in mice. J Surg Res 173: 332-340, 2012

6. Chang $\mathrm{CH}$, Kao $\mathrm{CH}$, Chio CC, Lin $\mathrm{CH}$, Lin MT and Chang CP: Attenuating heatstroke-induced acute lung inflammation, edema, and injury in rats by exercise preconditioning. J Trauma Acute Care Surg 74: 1052-1059, 2013.

7. Sun ML, Ma DH, Liu M, Yu YX, Cao DB, Ma C, Wang X and Liu XL: Successful treatment of paraquat poisoning by Xuebijing, an injection concocted from multiple Chinese medicinal herbs: A case report. J Altern Complement Med 15: 1375-1378, 2009.

8. Liu X, Hu Z, Zhou B, Li X and Tao R: Chinese herbal preparation Xuebijing protently inhibits inflammasome activation in hepatocytes and ameliorates mouse liver ischemia-reperfusion injury. PLoS One 10: e0131436, 2015.

9. Li GG and Han WB: Effect of Xuebijing on liver ischemia reperfusion injury. Modern Journal of Integrated Traditional Chinese and Western Medicine 18: 3282-3283, 2009 (In Chinese).

10. Qi F, Liang ZX, She DY, Yan GT and Chen LA: A clinical study on the effects and mechanism of Xuebijing injection in severe pneumonia patients. J Tradit Chin Med 31: 46-49, 2011.

11. Chen H, Li S and Zhu W: Protective effects of Xuebijing injection on lung injury in rats with sepsis. Journal of Internal Intensive Medicine 17: 91-92, 2011 (In Chinese).

12. Chen SH, Chang FM, Niu KC, Lin MY and Lin MT: Resuscitation from experimental heatstroke by estrogen therapy. Crit Care Med 34: 1113-1118, 2006.

13. Jiao LN, Yao YM and Shou ST: Effects of Xuebijing injection on hepatic gelsolin and inflammatory cytokine in septic rats. Medical Journal of Chinese People's Liberation Army 36: 14-16, 2011 (In Chinese).

14. Liu QQ, Zhu XQ and Wang L: Effects of Xuebijing injection on survival rats and liver-kidney function in rats with sepsis. Journal Of Emergency In Traditional Chinese Medicine 17: 203-205, 2008 (In Chinese).

15. Chen CM, Hou CC, Cheng KC, Tian RL, Chang CP and Lin MT: Activated protein $\mathrm{C}$ therapy in a rat heat stroke model. Crit Care Med 34: 1960-1966, 2006.

16. Chen Y, Tong H, Zhang X, Tang L, Pan Z, Liu Z, Duan P and Su L: Xuebijing injection alleviates liver injury by inhibiting secretory function of Kupffer cells in heat stroke rats. J Tradit Chin Med 33: 243-249, 2013.

17. Chen CC, Chen ZC, Lin MT and Hsu CC: Activated protein C improves heatstroke outcomes through restoration of normal hypothalamic and thermoregulatory function. Am J Med Sci 338: 382-387, 2009

18. Lin XJ, Li YL, Mei GP, Zou F, He DD, Liu XQ, Li YJ, Zhao TB and Lin MT: Activated protein $\mathrm{C}$ can be used as a prophylactic as well as a therapeutic agent for heat stroke in rodents. Shock 32: 524-529, 2009 .

19. Hong SB, Koh Y, Lee IC, Kim MJ, Kim WS, Kim DS, Kim WD and Lim CM: Induced hypothermia as a new approach to lung rest for the acutely injured lung. Crit Care Med 33: 2049-2055, 2005.

20. Su L: Review on prevention and treatment of severe heat stroke. Medical journal of Chinese People's Liberation Army 36: 883-885, 2011 (In Chinese).

21. Varghese GM, John G, Thomas K, Abraham OC and Mathai D: Predictors of multi-organ dysfunction in heatstroke. Emerg Med J 22: 185-187, 2005. 
22. Rinaldo JE, Dauber JH, Christman J and Rogers RM: Neutrophi alveolitis following endotoxemia. Enhancement by previous exposure to hyperoxia. Am Rev Respir Dis 130: 1065-1071, 1084

23. Yang HH, Chang CP, Cheng RT and Lin MT: Attenuation of acute lung inflammation and injury by whole body cooling in a rat heatstroke mode. J Biomed Biotechnol 2009: 768086, 2009.

24. Patel BV, Wilson MR, O'Dea KP and Takata M: TNF-induced death signaling triggers alveolar epithelial dysfunction in acute lung injury. J Immunol 190: 4274-4282, 2013.

25. Kunzmann S, Collins JJ, Kuypers E, Gavilanes AW and Kramer BW: Effects of antenatal inflammation on the developing lung. Z Geburtshilfe Neonatol 216: 177-185, 2012 (In German).

26. Pugin J, Ricou B, Steinberg KP, Suter PM and Martin TR: Proinflammatory activity in bronchoalveolar lavage fluids from patients with ARDS, a prominent role forinterleukin-1. Am J Respir Crit Care Med 153: 1850-1856, 1996.

27. Xing Z, Jordana M, Kirpalani H, Driscoll KE, Schall TJ and Gauldie J: Cytokine expression by neutrophils and macrophages in vivo: Endotoxin induces tumor necrosis factor-alpha, macrophage inflammatory protein-2, interleukin-1 beta, and interleukin- 6 but not RANTES or transforming growth factor-beta $1 \mathrm{mRNA}$ expression in acute lung inflammation. Am J Respir Cell Mol Biol 10: 148-153, 1994.

28. Zhu YB, Zhang YB, Liu DH, Li XF, Liu AJ, Fan XM, Qiao CH, Ling F and Liu YL: Atrial natriuretic peptide attenuates inflammatory responses on oleic acid-induced acute lung injury model in rats. Chin Med J (Engl) 126: 747-750, 2013.

29. Ma C, Zhu L, Wang J, He H, Chang X, Gao J, Shumin W and Yan T: Anti-inflammatory effects of water extract of Taraxacum mongolicum hand.-Mazz on lipopolysaccharide-induced inflammation in acute lung injury by Suppressing PI3K/Akt/ mTOR signaling pathway. J Ethnopharmacol 168: 349-355, 2015.
30. Wan LM, Tan L, Wang ZR, Liu SX, Wang YL, Liang SY, Zhong JB and Lin HS: Preventive and therapeutic effects of Danhong injection on lipopolysaccharide induced acute lung injury in mice. J Ethnopharmacol 149: 352-359, 2013.

31. Su CL, Du WY, Chiang LL, Lin YK, Lee HL, Chen KH, Wang J Jr and Wang D: Amelioration of superoxide dismutase on ventilator-induced lung injury by suppressing leukocyte in the lungs and systemic circulation. Chin J Physiol 56: 219-229, 2013.

32. Zhang L, Shen J, Gan ZY, He DK and Zhong ZY: Protective effect of melatonin in rats with phosgene-induced lung injury. Zhonghua Lao Dong Wei Sheng Zhi Ye Bing Za Zhi 30: 834-838, 2012 (In Chinese).

33. Malaviya R, Venosa A, Hall L, Gow AJ, Sinko PJ, Laskin JD and Laskin DL: Attenuation of acute nitrogen mustard-induced lung injury, inflammation and fibrogenesis by a nitric oxide synthase inhibitor. Toxicol Appl Pharmacol 265: 279-291, 2012.

34. Yen YT, Yang HR, Lo HC, Hsieh YC, Tsai SC, Hong CW and Hsieh $\mathrm{CH}$ : Enhancing autophagy with activated protein $\mathrm{C}$ and rapamycin protects against sepsis-induced acute lung injury. Surgery 153: 689-698, 2013.

35. Högner K, Wolff T, Pleschka S, Plong S, Gruber AD, Kalinke U, Walmrath HD, Bodner J, Gattenlöhner S, Lewe-Schlosser P, et al: Macrophage-expressed IFN- $\beta$ contributes to apoptotic alveolar epithelial cell injury in severe influenza virus pneumonia. PLoS Pathog 9: e1003188, 2013.

36. Liu WL, Liu ZW, Li TS, Wang C and Zhao B: Hydrogen sulfide donor regulates alveolar epithelial cell apoptosis in rats with acute lung injury. Chin Med J (Engl) 126: 494-499, 2013.

37. Sun YW, Niu TK, Yang JM, Kwon CH, Chen KY and Chen KM: Potentiation of the growth inhibiton activity of 2-(\{4-[4-(acridin-9-ylamino)phenylthio]phenyl\}(2-hydroxyethyl) amino)ethan-1-ol (CK0402) by Herceptin in SKBR-3 human breast cancer cells. Exp Ther Med 1: 513-518, 2010. 